\title{
Utilisation des figues de Barbarie en remplacement de la mélasse dans les blocs nutritionnels. Effets sur l'ingestion volontaire
}

\author{
Amor Chermiti* \\ Laboratoire de nutrition animale, Institut national de la recherche agronomique de Tunisie, \\ rue Hédi Karray, Ariana 2049, Tunisie
}

(Reçu le 25 juillet 1997 ; accepté le 10 mars 1998)

\begin{abstract}
Use of prickly pears to replace molasses in nutritional blocks. Effects on the voluntary intake. This study considered the chemical composition and the voluntary intake by heifers and ewes of feed blocks in which whole prickly pears were used to replace molasses. The animals were given feed blocks that contained either prickly pears or molasses, together with urea, wheat bran, cement as binder, and a commercial mixture of vitamins and minerals. They had free access to the blocks as well as to vetch-oat hay. The inclusion of urea in the blocks considerably increased their nitrogen contents, although this level was lower in the prickly pear based blocks. The voluntary intakes by both species were identical for both types of blocks: $0.82 \mathrm{~kg} \mathrm{DM} /$ day for heifers and $0.25 \mathrm{~kg} \mathrm{DM} /$ day for the ewes. The corresponding intakes of hay were $7.68 \mathrm{~kg} \mathrm{DM} /$ day and $1.02 \mathrm{~kg} \mathrm{DM} /$ day for the heifers and ewes, respectively. These results showed that prickly pears could be used to replace molasses in feed blocks. (1) Elsevier (Inra)
\end{abstract}

blocks / prickly pear / molasses / voluntary intake

Résumé - Ce travail étudie la composition chimique et l'ingestion volontaire des blocs alimentaires, chez des génisses et des brebis, à base de figues de Barbarie entières utilisées en remplacement de la mélasse. Les blocs sont constitués de mélasse ou de figues, d'urée, de son de blé, de ciment en tant que liant, et d'un mélange commercial de minéraux et de vitamines. Les animaux ont reçu à volonté les blocs en présence d'un foin de vesce - avoine distribué également à volonté. La présence d'urée dans la formule des blocs augmente considérablement leur teneur en azote, avec une teneur moins élevée dans les blocs à base de figues de Barbarie. Les quantités volontairement ingérées pour chaque espèce sont identiques entre les deux types de blocs et sont

\footnotetext{
* Correspondance et tirés à part

Tél. : (216) 1230 024/239; fax : (216) 1752897.
} 
en moyenne de $0,82 \mathrm{~kg} \mathrm{MS} \cdot \mathrm{j}^{-1}$ chez les génisses d'élevage et de $0,25 \mathrm{~kg} \mathrm{MS} \cdot \mathrm{j}^{-1}$ chez les brebis. Les quantités ingérées de foin sont respectivement de $7,68 \mathrm{~kg} \mathrm{MS} \cdot \mathrm{j}^{-1}$ et de $1,02 \mathrm{~kg} \mathrm{MS} \cdot \mathrm{j}^{-1}$. Les résultats montrent qu'il est possible de remplacer la mélasse par les figues de Barbarie entières dans les formules de blocs multinutritionnels. (@ Elsevier / Inra)

blocs / figues de Barbarie / mélasse / ingestion volontaire

\section{INTRODUCTION}

Les travaux visant la valorisation des sous-produits de l'agriculture et de l'industrie sont très nombreux et s'orientent depuis plusieurs années vers l'amélioration de leur valeur alimentaire, notamment pour ceux qui sont riches en lignocellulose tels que les pailles de céréales [3]. L'amélioration de la valeur alimentaire des sous-produits riches en lignocellulose et pauvres en azote consiste aussi à mettre à la disposition des animaux qui les reçoivent des blocs multinutritionnels apportant l'azote, les minéraux, et le minimum de glucides rapidement fermentescibles nécessaires tant à la couverture des besoins des ruminants qu'à l'optimisation de la cellulolyse et de la protéosynthèse des micro-organismes de leur rumen $[5,6,8]$. Un intérêt supplémentaire de ces blocs est de mettre à la disposition des animaux éloignés, les sous-produits rentrant dans leur composition sous une forme facile à transporter et à conserver.

Les premiers essais de fabrication de ces blocs ont été réalisés à partir de mélasse de betteraves et d'urée [7]. Dans les conditions tunisiennes, la mélasse produite est presque entièrement utilisée dans l'industrie de fabrication de levures utilisées dans les usines de pâtes alimentaires. La recherche d'autres produits pour remplacer la mélasse est d'un grand intérêt, notamment pour les éleveurs des zones arides et semi-arides où les ressources alimentaires sont limitées et fluctuantes d'une période à une autre.
L'objectif visé dans le présent travail est l'étude de la composition chimique et de l'ingestion volontaire des blocs à base de figues de Barbarie en remplacement de la mélasse chez les bovins et les ovins.

\section{MATÉRIEL ET MÉTHODES}

\subsection{Techniques de fabrication des blocs}

La technique de fabrication des blocs alimentaires est très simple, nécessite peu de moyens et est donc peu coûteuse. La procédure de fabrication est celle décrite par Sansoucy [7] et Garcia et Restrepo [4]. Il suffit de disposer d'une balance, d'un récipient où s'effectue le mélange ou un mélangeur dans le cas de fabrication de grandes quantités et d'un moule. Elle peut être réalisée même par des petits agriculteurs, disposant de peu de moyens. les matières premières sont mélangées manuellement une à une et la consistance du mélange est fonction de la proportion des substrats liquides.

Les matières premières utilisées dans cette ćtude sont la mélasse, l'urée, le son de blé, des minéraux et des vitamines et du ciment, utilisé comme liant. Les proportions des différents ingrédients sont respectivement de $40 \%$, $10 \%, 35 \%, 5 \%$ et $10 \%$. Les figues de Barbarie entières, après leur broyage, ont été substituées à la mélasse. Les blocs à base de mélasse sont utilisés comme témoin.

\subsection{Analyses chimiques}

Les échantillons représentatifs des blocs, séchés à $50^{\circ} \mathrm{C}$ et broyés avec un broyeur à marteau portant une grille de $1 \mathrm{~mm}$, ont été analysés pour déterminer les teneurs en matière 
sèche, matière organique, matières azotées totales et en cellulose brute selon les techniques de l'AOAC [1].

\subsection{Ingestion volontaire}

Quatre génisses de type croisé (Locale*Pie Noire) et quatre brebis adultes de race Barbarine, placées en cage individuelle sans litière, ont reçu à volonté les blocs à base de mélasse ou de figues de Barbarie en présence de foin de vesce-avoine, distribué également à volonté. Le poids moyen des génisses en début d'expérience est de $294 \mathrm{~kg}$ et celui des brebis est de $57 \mathrm{~kg}$. Les blocs étaient à la disposition des animaux durant toute la journée et le foin était distribué en trois repas. Les quantités volontairement ingérées de blocs et de foin ont été mesurées par pesée quotidienne des quantités distribuées et refusćes pendant 15 à $20 \mathrm{j}$, après une période d'adaptation de deux semaines. La matière sèche des aliments a été déterminée une fois par semaine et les animaux pesés tous les 15 jours.

\subsection{Analyses statistiques}

Les données ont été soumises à l'analyse de la variance en utilisant le logiciel STTAT.ITCF et les différences entre les moyennes ont été calculées en utilisant le test de Newman-Kulls.

\section{RÉSULTATS ET DISCUSSION}

\subsection{Composition chimique des blocs}

Le foin de vesce-avoine utilisé dans cet essai à une teneur en matières azotées totales de $7,1 \%$ de la MS et une teneur en cellulose brute de $37,2 \%$ (tableau I). Il correspond donc à un foin de qualité moyenne dans les conditions tunisiennes. La teneur en matière organique des blocs est de $79,5 \%$ et de $62,8 \%$, respectivement pour ceux à base de mélasse et de figues de Barbarie (tableau I). La faible teneur en matière organique des blocs à base de figues est liée à la teneur élevée des figues en matières minérales, notamment en phosphore et en potassium (A. Chermiti, non publié). Ceci suggère que la complémentation de ces types de blocs en minéraux doit être appropriée. La teneur élevée en matières azotées des blocs résulte de l'incorporation de $10 \%$ d'urée dans le mélange. Cependant, cette teneur est moins élevée avec les blocs à base de figues. Il est à noter que les broyats de figues présentent une teneur moyenne en matières azotées totales de seulement $5.5 \%$ de la matière sèche (A. Chermiti, non publié). Il est possible que l'urée, en présence de figues, ait été partiellement transformée en ammoniac d'où une proportion moins importante en azote avec ce type de blocs. D'ailleurs, une odeur d'ammoniac se dégage de ces blocs quelques jours après leur fabrication. Il nous faudra dans l'avenir analyser les différentes fractions d'azote dans ces blocs. La teneur en cellulose brute est deux fois plus élevée dans les blocs à base de figues de Barbarie que dans ceux à base de mélasse. La présence de pépins dans les figues est probablement à l'origine de cette différence. D'ailleurs, la teneur en cellu-

Tableau I. Composition chimique (en \% de la MS) des aliments.

\begin{tabular}{lccrr}
\hline Aliments & MS & MO & MAT & \multicolumn{1}{c}{ CB } \\
\hline Foin de vesce-avoine & 87,7 & 93,3 & 7,1 & 37,2 \\
Bloc à base de mélasse & 82,5 & 79,5 & 40,2 & 5,1 \\
Bloc à base de figues & 74,9 & 62,8 & 25,2 & 11,1 \\
\hline
\end{tabular}


lose brute des figues de Barbarie entières est en moyenne de $20 \%$ (A. Chermiti, non publié).

\subsection{Quantité volontairement ingérées}

Les résultats montrent que, pour chaque espèce, les quantités volontairement ingérées de blocs et de foin sont similaires (tableau II). Les génisses ingèrent en moyenne $8,5 \mathrm{~kg}$ de matière sèche par jour (7,68 $\mathrm{kg}$ de foin et $0,82 \mathrm{~kg}$ de blocs) et les brebis ingèrent $1,27 \mathrm{~kg}$ de matière sèche par jour $(1,02 \mathrm{~kg}$ de foin et $0,25 \mathrm{~kg}$ de blocs). Des quantités ingérées de blocs à base de mélasse et d'urée variant de 0,35 à $0,57 \mathrm{~kg} \cdot \mathrm{j}^{-1}$ ont été rapportées par Allen [2] chez les vaches locales croisées alimentées avec une ration à base de fourrages verts.

Il est à noter que les quantités volontairement ingérées des blocs à base de figues ou de mélasse ont été variables et plus particulièrement chez les génisses (figure 1). Elles peuvent atteindre environ $2 \mathrm{~kg}$ par jour chez les génisses et $500 \mathrm{~g}$ par jour chez les brebis, soit respectivement des ingestions de $200 \mathrm{~g}$ et de $50 \mathrm{~g}$ d'urée par jour et par animal (l'urée représentait $10 \%$ dans le mélange). Ces ingestions élevées en urée ne sont pas toxiques étant donné que les blocs sont ingérés tout au long de la journée et non en un temps limité comme c'est le cas des aliments concentrés. Il devrait en résulter une répartition normale de la concentration ammoniacale dans le contenu ruminal au cours de la journée, mais cela reste à confirmer par l'étude des paramètres de la fermentation dans le rumen.

\section{CONCLUSIONS ET INTÉRÊTS PRATIQUES}

La présente étude montre qu'il est possible de remplacer totalement la mélasse par des figues de Barbarie dans la fabri-

Tableau II. Quantités volontairement ingérées de foin de vesce-avoine et de blocs chez des génisses d'élevage et des brebis adultes.

\begin{tabular}{llcc}
\hline Animaux & \multicolumn{1}{c}{ Rations } & MS/j $(\mathrm{kg})$ & $\mathrm{MS}^{\prime} \mathrm{kg} \mathrm{P} \mathrm{P}^{0,75}(\mathrm{~g})$ \\
\hline Génisses & Foin + blocs mélasse & $8,73 \pm 1,3$ & $127 \pm 19$ \\
& Foin & $7,85 \pm 1,2$ & $114 \pm 15$ \\
& Bloc & $0,88 \pm 0,2$ & $13 \pm 3,7$ \\
& Foin + blocs figues & $8,27 \pm 0,7$ & $120 \pm 18$ \\
& Foin & $7,51 \pm 0,7$ & $109 \pm 16$ \\
& Bloc & $0,76 \pm 0,09$ & $11 \pm 1,6$ \\
& & \\
Brebis & Foin + blocs mélasse & $1,27 \pm 0,15$ & $60 \pm 7,3$ \\
& Foin & $1,02 \pm 0,16$ & $49 \pm 5,5$ \\
& Bloc & $0,24 \pm 0,02$ & $11 \pm 1,0$ \\
& Foin + blocs figues & $1,28 \pm 0,33$ & $59 \pm 18$ \\
& Foin & $1,02 \pm 0,28$ & $46 \pm 1,3$ \\
& Bloc & $0,26 \pm 0,06$ & $13 \pm 2,4$
\end{tabular}

Les différences constatées pour chaque espèce entre l'ingestion des deux blocs ne sont pas significativement différentes $(P<0,05)$. 

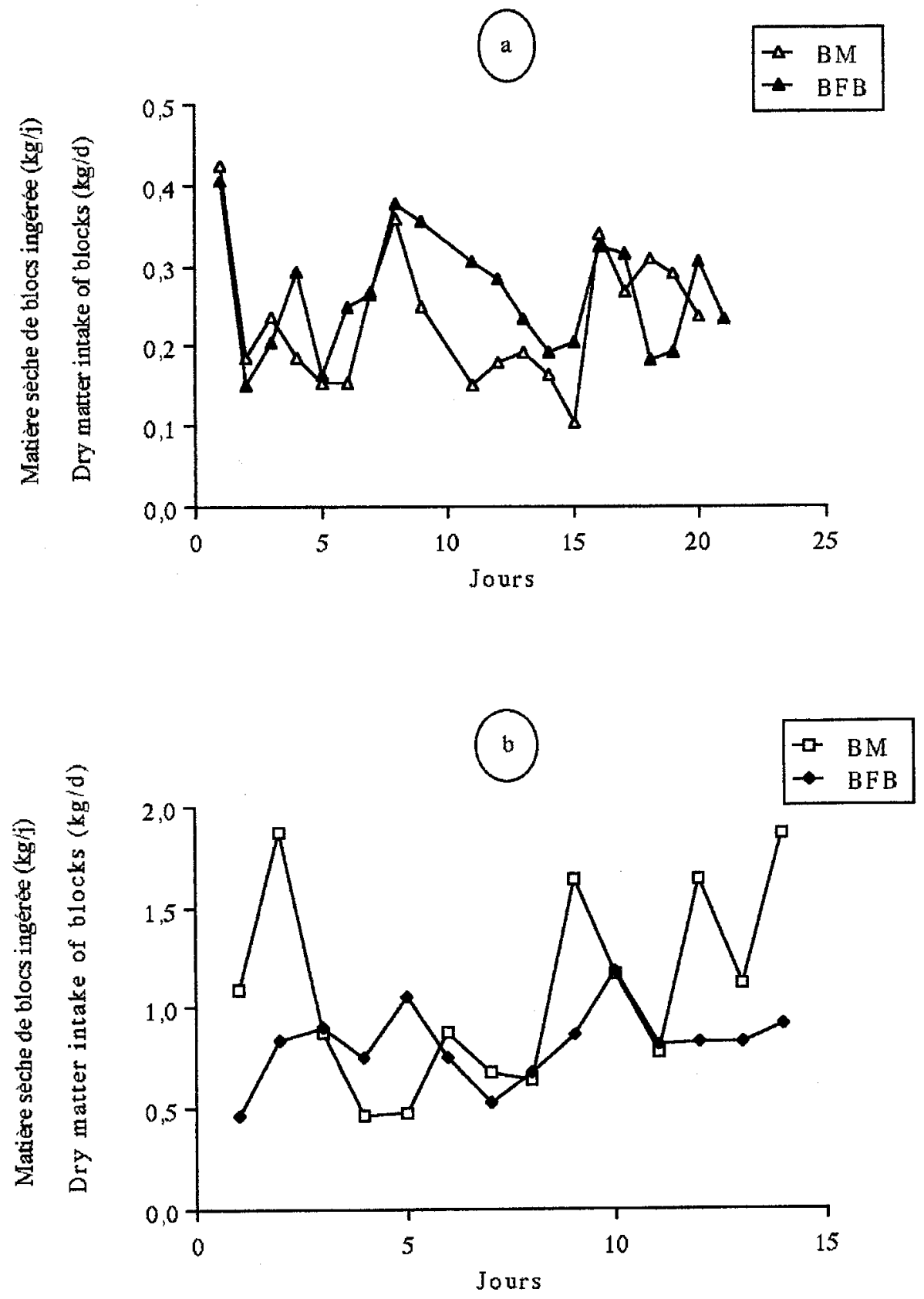

Figure 1. Variation des quantités moyennes journalières ingérées de matière sèche des blocs à base de mélasse (BM) ou de figues de Barbarie (BFB) chez des brebis adultes (a) et des génisses d'élevage (b). 
cation des blocs multinutritionnels. Les figues de Barbarie provenant des plantations de cactus chez les petits éleveurs des zones arides et semi-arides peuvent être incorporées dans ces blocs en association avec d'autres sous-produits tels que le son de blé et les pulpes de tomates. Ces blocs se conservent sans problème et pendant plus d'une année. Ils présentent un grand intérêt pour la complémentation des fourrages grossiers, des parcours et des chaumes de céréales utilisés pendant les mois d'été et d'automne et constituent pour les ruminants une source alimentaire importante et stratégique pour les zones arides et semi-arides. Les blocs alimentaires à base de figues de Barbarie sont bien ingérés par les animaux. Leur ingestion, étalée tout au long de la journée, présente l'avantage de fournir aux microorganismes du rumen des conditions très favorables à leur prolifération. La présence en quantités suffisantes dans ces blocs d'urée et de sucres rapidement fermentescibles constitue une source appréciable d'énergie et d'azote pour la flore ruminale.

La fabrication des blocs multinutritionnels à base de figues de Barbarie et d'urée présente de nombreux avantages : i) la conservation de plusieurs sous-produits pendant de longues périodes sans que la valeur alimentaire soit atteinte; ii) le transport facile d'une région à une autre et en grandes quantités; et iii) l'utilisation en tant que compléments aux fourrages pauvres et aux parcours.

\section{REMERCIEMENTS}

Nous tenons à remercier Monsieur C. Demarquilly, directeur de l'unité de la valeur alimentaire à Theix, pour les suggestions et les conseils judicieux qu'il a apportés au manuscrit.

\section{RÉFÉRENCES}

[1] AOAC, Official Methods of Analysis of the Association of Official Analytical Chemists, 12th edn., Washington, D.C., 1975.

12] Allen M.L., Making molasses-urea feedblocks, Asian Livestock. (1986), 154-166.

[3] Chermiti A., Utilisation des pailles de céréales traitées à l'ammoniac et à l'urée par différentes espèces de ruminants dans les pays d'Afrique du Nord, Thèse de Doctorat en Sciences Agronomiques, Université Catholique de Louvain, Louvain-la-Neuve, Belgique, 1994, 214 p.

[4] Garcia L.O., Restrepo J.I.R., Multinutrient block handbook. FAO, Better Farming Series, 45 (1985) 1-18.

[5] Kakkar V.K., Kaur S., The value of ureamolasses-liquid diets in ruminants. A review, Agric. Rev. 14 (1993) 109-120.

[6] Leng R.A., The potentiel of solidified molasses based blocks for the correction of multinutritionnal deficiencies in buffaloes and other ruminants fed low-quality agroindustrial by-products. In the use of the nuclear techniques to improve domestic buffalo production in Asia, Vienna, Austria, International Atomic Energy Agency, 1984, pp. 135-149.

[7] Sansoucy R., New development in the manufacture and utilization of multinutrient blocks, World Anim. Rev. 82 (1995) 78-83.

[8] Tamboura H., Abou F., Utilisation des blocs de mélasse-urée pour la valorisation des pailles de riz dans l'alimentation des moutons en pays tropicaux sahéliens, Bull. Anim. Prod. Afr. 40 (1992) 25-31. 